http://dx.doi.org/10.35381/e.k.v4i8.1359

\title{
Brecha digital en tiempos de pandemia: Perspectivas de padres de familia
}

Digital divide in times of pandemic: Perspectives of parents of family

\author{
Diana Gabriela Bermeo-Chalco \\ diana.bermeo.05@est.ucacue.edu.ec \\ Universidad Católica de Cuenca, Azogues \\ Ecuador \\ https://orcid.org/0000-0003-2350-8440 \\ Darwin Gabriel García-Herrera \\ dggarciah@ucacue.edu.ec \\ Universidad Católica de Cuenca, Azogues \\ Ecuador \\ https://orcid.org/0000-0001-6813-8100 \\ Sandra Elizabeth Mena-Clerque \\ sandramena@ucacue.edu.ec \\ Universidad Católica de Cuenca, Cuenca \\ Ecuador \\ https://orcid.org/0000-0002-9186-2161
}

Recepción: 15 de marzo 2021

Revisado: 15 de mayo 2021

Aprobación: 15 de junio 2021

Publicación: 01 de julio 2021 


\title{
RESUMEN
}

La pandemia ha obligado a cerrar instituciones educativas y a emplear otras formas de enseñar cómo es la educación virtual. Sin embargo, no todos han tenido las mismas oportunidades, debido a que existe una desigualdad tecnológica. Es por ello, que la investigación tiene como objetivo identificar la brecha digital en la educación, dificultad generada en tiempos de pandemia COVID-19 desde la percepción de los padres de familia del subnivel inicial del Cantón Gualaceo. Para ello, el trabajo fue desarrollado desde una tipología descriptiva no experimental y de cohorte transversal, apoyada en un enfoque mixto, mediante la aplicación de una encuesta y un grupo focal, identificándose la dificultad principal que es la brecha digital de uso de la tecnología, es decir, la falta de conocimiento o bajo nivel de competencias digitales de los representantes legales, inconveniente que afecta el apoyo directo del aprendizaje de los niños en sus hogares.

Descriptores: Aprendizaje activo; método de aprendizaje; aprendizaje en línea. (Palabras tomadas del Tesauro UNESCO).

\begin{abstract}
The pandemic has forced the closure of educational institutions and the use of other ways of teaching what virtual education is like. However, not everyone has had the same opportunities, due to technological inequality. That is why the research aims to identify the digital gap in education, a difficulty generated in times of the COVID-19 pandemic from the perception of the parents of the initial sublevel of the Gualaceo Canton. For this, the work was developed from a descriptive, non-experimental and cross-sectional cohort typology, supported by a mixed approach, through the application of a survey and a focus group, identifying the main difficulty, which is the digital gap in the use of technology, that is, the lack of knowledge or low level of digital skills of legal representatives, an inconvenience that affects the direct support of children's learning at home.
\end{abstract}

Descriptors: Activity learning; learning methods; electronic learning. (Words taken from the UNESCO Thesaurus). 


\section{INTRODUCCIÓN}

En el año 2020 a nivel mundial se empezó a enfrentar a la COVID-19, una pandemia que produjo impactos relevantes en diferentes ámbitos como económicos, sociales, políticos, religiosos, culturales y por supuesto educativos. Es así que, en el ámbito educativo, la mayoría de países tuvieron que cerrar transitoriamente las instituciones. Esto afectó a más del $91 \%$ de estudiantes, por cuanto alrededor de 1600 millones de alumnos en el mes de abril estaban fuera de sus escuelas y colegios como lo menciona la (Organización de Naciones Unidas para la Educación, Ciencia y la Cultura, UNESCO, 2020a).

En realidad, la educación enfrenta diferentes dificultades a partir de la pandemia y sin duda las Tecnologías de la Información y Comunicación (TIC) se han convertido en un eje importante, y una gran ayuda para que la educación continúe con su propósito de enseñanza. Sin embargo, su implementación en las aulas de clases fue imprevisto, generando una variedad de cambios en toda la comunidad educativa. Debido a que, toda actividad o entorno se volvió digital produciendo una desigualdad o desequilibrio denominado brecha digital.

Cabe señalar que, esta diferencia ya existía desde antes de la pandemia, es decir, existía ya una brecha digital visible y dividida de dos maneras, la una internacional, que se da entre los países ricos y los pobres, y la otra internacional que surge en cada país como lo señala, (Rodríguez-Gallardo, 2006). Aunque es una problemática de algún tiempo atrás, ha resaltado en el año 2020 afectando a toda la población y a todos los contextos, sobre todo en el ambiente educativo, como una desigualdad digital para no poder avanzar.

En efecto, la brecha digital es un problema que causa desigualdad, debido a las diferencias que existen en acceder a los beneficios de la tecnología. Sin duda, esta dificultad afecta a diferentes países internacionales como España, donde según estudios, aunque el alumnado cuente con dispositivos electrónicos en un $96,7 \%$ y una conexión a internet de $83,6 \%$ en los hogares, no ha sido suficiente, debido a que, no es lo mismo tener tecnología que saber de competencias digitales, por lo que, los estudiantes de 
EPISTEME KOINONIA

Revista Electrónica de Ciencias de la Educación, Humanidades, Artes y Bellas Artes

Año IV. Vol IV. N 8 . Julio - Diciembre. 2021

Hecho el depósito de Ley: FA2018000022

ISSN: 2665-0282

FUNDACIÓN KOINONIA (F.K)

Santa Ana de Coro, Venezuela

Diana Gabriela Bermeo-Chalco; Darwin Gabriel García-Herrera; Sandra Elizabeth Mena-Clerque

España se han visto afectados por esta situación como lo exponen (Rodicio-García et al. 2020).

De una forma similar sucede en África, por la pandemia las escuelas cerraron, lo que empeoró más el entorno desfavorecedor que ya existía y aún más a los alumnos vulnerables. Sin embargo, no solo afectó a ellos, sino también a sus representantes que tomaron un rol de educador en sus hogares, pero debido a su bajo nivel de instrucción y por su baja economía no pueden ayudar a sus hijos en su totalidad, además, los docentes también se han visto afectados.

En África solo el $64 \%$ de profesores han recibido una formación que no incluye competencias digitales, aptitudes básicas en tecnologías de la información y la comunicación - TIC, lo que dificulta impartir y recibir clases virtuales de calidad como lo indica la Organización de Naciones Unidas para la Educación, Ciencia y la Cultura, UNESCO, 2020b).

En cuanto a Ecuador, aunque las telecomunicaciones hayan tenido un fuerte crecimiento durante los últimos años, la brecha digital en la población aún perdura. Además, en las diferentes encuestas realizadas se confirmó que no existe un fuerte nivel tecnológico, debido a factores socioeconómicos que está afectando directamente al acceso a la tecnología (Ministerio de Telecomunicaciones y de la Sociedad de la Información, MINTEL 2019). Por lo tanto, esto no permite facilitar los procesos de enseñanza aprendizaje a un nivel competitivo, considerando que las TIC constituyen una herramienta fundamental para mejorar el proceso educativo.

Por esta razón, la presente investigación tiene como objetivo identificar de cerca la existencia de la brecha digital en el cantón Gualaceo, específicamente en la escuela Mercedes Vázquez Correa, en virtud de que, no se han realizado estudios relacionados a este tema. El propósito investigativo es mostrar su incidencia desde la percepción de padres de familia, quienes han adoptado un nuevo rol y estilo de enseñanza - aprendizaje durante la pandemia. De tal modo que, se pueda indagar qué dificultades tienen en el acompañamiento a sus representados con las clases virtuales. 
EPISTEME KOINONIA

Revista Electrónica de Ciencias de la Educación, Humanidades, Artes y Bellas Artes

Año IV. Vol IV. Nº. Julio - Diciembre. 2021

Hecho el depósito de Ley: FA2018000022

ISSN: 2665-0282

FUNDACIÓN KOINONIA (F.K)

Santa Ana de Coro, Venezuela

Diana Gabriela Bermeo-Chalco; Darwin Gabriel García-Herrera; Sandra Elizabeth Mena-Clerque

\section{Referencial teórico}

\section{La educación en tiempos de pandemia}

Las recientes investigaciones demuestran que la educación ha cambiado drásticamente gracias a la pandemia denominada COVID-19 que estamos enfrentando hasta la fecha. Cabe señalar, que son cambios que ha puesto a prueba no solo a docentes, estudiantes, sino también a padres de familia o representantes legales. En efecto, la pandemia ha sido causa de cambios directos en el campo educativo, ya que, por medidas de seguridad las instituciones educativas cerraron, convirtiéndose en clases virtuales y valiéndose de los dispositivos electrónicos y recursos tecnológicos.

En pocas palabras, la educación al ser un derecho fundamental de los niños y los jóvenes no podía suspenderse, por lo que, se adoptaron diferentes estrategias para continuar con los estudios. Sin embargo, fue un cambio repentino que no ha favorecido a todos por igual, teniendo como resultado un aprendizaje desigual. Dicho de otra manera, la educación tuvo un gran impacto en muchos países, tal es el caso de Chile, el acceso a internet en el país y la cobertura comunal es desigual. Aunque se han entregado computadoras a los estudiantes que pertenecen al $40 \%$ más pobre, no es suficiente para continuar con la educación online (Quiroz-Reyes, 2020).

Es más, no es el único problema que afronta, ya que, las diferencias del grupo familiar también se evidencian. Dado que, las instituciones han delegado importante responsabilidad del proceso educativo a las familias, lo que convierte en una desventaja para los estudiantes de los sectores socioeconómicos bajos, en vista de que, si antes ya era difícil apoyar a sus hijos, ahora aún más porque muchos desconocen de una comprensión digital.

En cuanto a Ecuador, también tuvo que adaptarse al cambio, ya que mediante el Acuerdo Nro.00013-A del 12 de marzo se dispuso de manera obligatoria la suspensión de clases en todas sus instituciones y modalidades. Esto produjo la aplicación del Plan Educativo Aprendamos Juntos en Casa, que consta de una herramienta y plataforma colectiva para desarrollarla desde los hogares, elaborada con el objetivo de disponer con una variedad 
de materiales y recursos educativos para poderla trabajar de manera conjunta con estudiantes, padres de familia y docentes (Ministerio de Educación MINEDUC, 2020). Ahora bien, a pesar haber aplicado diferentes estrategias y recursos educativos, en los últimos reportes registrados existen tres millones de estudiantes de escuelas fiscales, de los cuales solo dos millones están teniendo conectividad y acceso a plataformas educativas, pero el millón restante no corre con la misma suerte, en razón de que no disponen de un computador, un teléfono, internet, ni tampoco conocimiento sobre su uso, como lo indica el Ministerio de Telecomunicaciones y de la Sociedad de la Información MINTEL, 2020), por lo que se han propuesto dos caminos, la una trabajar en procesos de conectividad y la otra mediante la televisión.

En cierto modo, la pandemia aceleró el proceso de digitalización y evidenció diversas carencias que tiene el ámbito educativo, debido a que las aulas de clases tuvieron que ser trasladas a las casas. Lo que ha traído una serie de cambios, entre ellos se encuentra una transformación significativa en los hogares y la necesidad de disponer de la tecnología como fuente principal para avanzar. Muchas familias de diferentes países sufrieron en mayor o menor medida, pero fue menos la impresión para países avanzados en tecnología, que los países que estaban en un proceso de adaptación tecnológica, pues esas familias tienen problemas todavía en adaptarse.

Sin duda, la educación ha enfrentado diferentes dificultades, por ende, también toda su comunidad educativa. Debido a que, las actividades escolares ya no son solo responsabilidad del estudiante sino también de sus representantes. Hay que hacer notar, que en todos los niveles escolares y en cualquier modalidad de estudio se necesita la colaboración de los padres de familia. Sin embargo, a los estudiantes del subnivel Inicial se vuelve más complicado todavía, debido a que, ellos necesitan la supervisión constante de un adulto en todas las acciones.

Por consiguiente, el representante legal de un niño de Inicial, a más de buscar un espacio para recibir las clases virtuales, ingresar y acompañar a las clases mediante códigos, supervisar e inclusive enseñar a la vez, preparar portafolios, grabar videos, audios, 
aprender a utilizar plataformas para enviar los deberes, eso mezclado con las responsabilidades del hogar, es lo que tuvo que enfrentar para no estancar la educación de su hijo.

En resumen, la educación a partir de la pandemia enfrenta varios cambios y con ella también los docentes, estudiantes y padres de familia. Los últimos asumiendo un rol con más responsabilidad para adaptarse y ayudar a sus hijos a las nuevas formas de aprender e interactuar desde sus hogares, en cierto modo, también se convierte en más responsabilidad y un reto para el docente, pues debe fortalecer sus capacidades para inclusive dar acompañamiento no solo al estudiante sino también a su representante, para que ellos a la vez puedan brindar ayuda y aprendizaje a sus hijos. Puesto que, a la edad de 4 años, un niño necesita la ayuda no solo del docente, sino también de sus familiares para el desarrollo afectivo, físico, social y cognitivo (Castillo-Fuerman, 2020).

\section{Brecha digital}

En un inicio brecha digital fue conceptualizada de manera incierta y ambigua (Gunkel, 2003); es decir, se desconoce cómo, de quién y cuándo apareció el término. Sin embargo, brecha digital es uno de los fenómenos más discutidos, claros y confusos de nuestros tiempos como lo menciona (Warschauer, 2001). Por lo que, se caracteriza como varios fenómenos diferentes; primero, la desigualdad al acceso y el uso de internet y segundo, la desigualdad para usar el internet, haciendo alusión a factores como educación, lenguaje y contenido. Pese a esto, la brecha digital se define como una estratificación social, ya que existe una desigualdad en la capacidad para acceder, adaptar y crear conocimientos mediante el uso de información y tecnologías de la comunicación.

La brecha digital también se origina con diferentes factores: primero, el nivel socioeconómico. Pues en un principio el factor económico era una barrera, ya que, los equipos tecnológicos y la conexión a internet eran escasas. El segundo factor es el nivel de formación de las personas, entendiendo como importante la capacidad intelectual para seleccionar información y obtener resultados. 
El tercer factor es geográfico, ya que hay diferencias entre zonas, tanto urbanas como rurales o entre otros países. El cuarto factor es la lengua, dado que el inglés domina la red, lo cual facilita su uso y la utilidad. El quinto factor es el sexo, debido a que, es muy baja la participación de las amas de casa en la sociedad de la información. Y el sexto factor es la edad, ya que, existe una distancia entre los nativos digitales y los inmigrantes digitales en cuanto a la utilización de la tecnología (Martín-Romero, 2020).

Es importante mencionar, que, aunque la brecha digital se fase en diferentes factores como la de acceso, la accesibilidad ha ido disminuyendo día con día, debido a que, en esta actualidad es muy raro ver a una persona sin disponer de un dispositivo electrónico, con una variedad en calidad, pero es un elemento que está desvaneciendo poco a poco. En cambio, su uso y calidad de utilidad sigue persistiendo convirtiéndose en exclusión entre los que saben utilizar en su vida cotidiana y se benefician de ello con los que no lo usan de manera básica, ya que no saben cómo hacerlo o simplemente no quieren hacerlo. Cabe resaltar, que la brecha digital es un problema que están teniendo diferentes países, ha crecido con la nueva modalidad de vida, conduciendo a una exclusión digital entre la población, debido a que aproximadamente un tercio de los hogares carecen de internet y sin conocer su utilidad; es decir, no se trata solo de tener o no acceso, también se trata de una habilidad y aprendizaje dividido (Stingl, 2016).

Es importante recalcar, que la brecha digital durante la pandemia ha afectado en gran medida a la educación de muchos niños y niñas, incluso ha sido forma de abandono escolar, debido a que no hay acceso a internet, aparatos electrónicos, dificultades en accesibilidad y los padres no son alfabetizados para apoyar a sus hijos, por lo que prefieren, descansar un año escolar hasta que todo vuelva a la normalidad (El Comercio, 2020). Por lo tanto, existe una diferencia, pero no solo en sectores rurales y urbanos, sino en todo el país, donde se experimenta la falta de acceso y uso de las TIC para desarrollar las nuevas formas de aprendizaje como lo menciona (Mosquera, 2020). 


\section{Competencias digitales}

La brecha digital abarca no solo al acceso a internet, sino también a su utilización, habilidad y conocimiento. Es decir, poniendo énfasis a las competencias digitales que son las que facilitan la interacción y aprendizaje de manera eficaz de diferentes actividades por medio del uso a los dispositivos electrónicos, aplicaciones y recursos tecnológicos. Además, estas competencias permiten sacar provecho de las TIC para poderlo emplear satisfactoriamente y obtener beneficios favorables como forma de progreso. Por lo tanto, es necesario mencionar que el desconocimiento y utilización a las TIC puede afectar no solo al ámbito educativo, sino social, económico y laboral como lo señala la (Organización de Naciones Unidas para la Educación, Ciencia y la Cultura, UNESCO, 2018).

Por consiguiente, al existir un bajo nivel en competencia digital seguirá existiendo esa brecha entre las personas que saben más y las que desconocen el uso de la tecnología. Pues la solución no está en incorporar cada vez más tecnología, ya sea en un aula de clases, en el trabajo, país, ciudad, sino formar a la ciudadanía para que pueda utilizarla correctamente y en beneficio de muchos, sin tomar en cuenta, que sean de distinto nivel social, género, edad, estructura familiar, cultura o nivel educativo, se debería instruir a todos porque solo así disminuirá la alfabetización digital e irá desapareciendo esa brecha que aún se vive en la mayoría de los países como forma de exclusión.

\section{El aprendizaje en el hogar}

A pesar de las dificultades a causa de la pandemia los padres de familia se convirtieron en guías y apoyos directos de los estudiantes. Es evidente, que ellos no son docentes, desconocen de herramientas pedagógicas, asignaturas, estrategias y aunque viven con una gran cantidad de preocupaciones tanto en salud, economía y familiar se han integrado para dar continuidad a los estudios de sus hijos e hijas y contribuir al aprendizaje (El Fondo de las Naciones Unidas para la infancia UNICEF, 2020). 
Por esta razón, al estar el padre de familia implicado en la educación del niño es muy importante su nivel educativo o preparación constante para obtener el éxito escolar de sus hijos, puesto que, son ellos los que ahora están involucrados en las nuevas actividades de las clases virtuales, es decir, preparados o no, ellos están asumiendo el papel de educador realizando esfuerzos grandes para apoyar a los niños, aunque no cuenten con la preparación académica necesaria.

Surge la preocupación en la mayoría de ellos la falta de preparación que les impide ayudar a sus hijos con éxito en las actividades encomendadas por los docentes. Es más, el nivel de preocupación y malestar es aún más alto en representantes de niños pequeños de entre 0 a 7 años, que asumen mayor responsabilidad y carga en apoyar la educación a distancia (Alpizar et al. 2020).

\section{METODOLOGÍA}

Esta investigación fue desarrollada desde una tipología descriptiva no experimental tranversal, desde el enfoque mixto, con la intención de recopilar información cuantitativa y cualitativa con la intención de estudiar el tema de estudio desde diversos ángulos investigativos. Se trabajó con una muestra poblacional de 30 representantes legales de la escuela fiscal Mercedes Vázquez Correa perteneciente al cantón Gualaceo, provincia del Azuay - Ecuador, para poder de esta manera indagar detalladamente la existencia de la brecha digital en el nivel inicial.

En cuanto a la técnica utilizada para la recolección de datos fue la encuesta, cuyo objetivo es "obtener y elaborar datos de modo rápido y eficaz" como lo manifiesta (Casas-Anguita, 2003, p. 143), la misma que permitió desarrollar y tener al alcance la mayor información posible. Como instrumento fue el cuestionario, misma que constaba de 10 preguntas en escala de Likert, basada en respuestas alternativas, de manera online y validada a través del coeficiente Alfa de Cronbach con un valor de 0,754 de fiabilidad, resultando aceptable para el estudio. Igualmente, todos los datos y tablas fueron procesados con ayuda del programa de estadística SPSS para poder obtener información confiable. 
Complementando a la investigación, se empleó un grupo focal compuesto por 6 participantes, los que debieron provenir de un mismo contexto, en este caso, representantes legales del nivel inicial, quienes fueron entrevistados para poder identificar con mayor detalle la temática abordada.

\section{RESULTADOS}

Los resultados adquiridos de la encuesta se realizaron utilizando una estadística descriptiva del SPSS. Además, se realizó la prueba de normalidad Shapiro Wilk, donde al analizarla se demostró que todas las variables son paramétricos, presentando de esta manera un valor menor a 0,05 sig.

\section{Tabla 1.}

La educación tuvo dificultad en la pandemia.

\begin{tabular}{llrrrr}
\hline & & & Porcentaje & Porcentaje \\
& & Frecuencia & Porcentaje & válido & acumulado \\
\hline \multirow{2}{*}{ Válidos } & Totalmente de acuerdo & 7 & 23,3 & 23,3 & 23,3 \\
& Bastante de acuerdo & 21 & 70,0 & 70,0 & 93,3 \\
& De acuerdo & 2 & 6,7 & 6,7 & 100,0 \\
\cline { 2 - 6 } & Total & 30 & 100,0 & 100,0 & \\
\hline
\end{tabular}

Fuente: Encuesta.

Del total de encuestados, el $6 \%$ está de acuerdo, el $70 \%$ bastante de acuerdo y $23 \%$ totalmente de acuerdo de que la educación tuvo dificultades en tiempos de pandemia. Por lo tanto, se puede determinar que la población analizada evidencia que la educación tuvo que enfrentar diferentes problemas y cambios inesperados para desarrollar la enseñanza - aprendizaje. 


\section{Tabla 2.}

Falta de capacitación.

\begin{tabular}{llrrrr}
\hline & & & & & \multicolumn{2}{c}{$\begin{array}{c}\text { Porcentaje } \\
\text { acumulado }\end{array}$} \\
\hline \multirow{4}{*}{ Válidos } & Demasiado & 4 & 13,3 & 13,3 & 13,3 \\
& Mucho & 17 & 56,7 & 56,7 & 70,0 \\
& Poco & 9 & 30,0 & 30,0 & 100,0 \\
\cline { 2 - 6 } & Total & 30 & 100,0 & 100,0 & \\
\hline
\end{tabular}

Fuente: Encuesta.

Como se mencionó anteriormente, uno de los cambios ocasionados a partir de la pandemia fue la educación virtual, lo que obligó a estudiar desde los hogares, dando uso de las competencias digitales. Sin embargo, como se puede observar en la tabla con la información de la población analizada, tiene como resultado, que el 30\% de encuestados está de acuerdo que la falta de capacitación es un factor que afecta poco, el 56\% contesta que afecta mucho y $13 \%$ contesta que afecta demasiado. Esto quiere decir, que no todos se sienten capacitados para colaborar en la educación virtual de sus hijos.

Tabla 3.

Relación entre el nivel de formación y el conocimiento al uso de las TIC.

\begin{tabular}{|c|c|c|c|c|c|c|c|}
\hline & & \multicolumn{5}{|c|}{ Nivel de formación } & \multirow[b]{4}{*}{ Total } \\
\hline & & \multirow[b]{3}{*}{ Ninguno } & \multicolumn{2}{|l|}{ Educación } & \multirow{3}{*}{$\begin{array}{c}\text { Tercer } \\
\text { nivel }\end{array}$} & \multirow{3}{*}{$\begin{array}{c}\text { Cuarto } \\
\text { nivel }\end{array}$} & \\
\hline & & & General & & & & \\
\hline & & & Básica & Bachillerato & & & \\
\hline Nivel de & Nada & 0 & 2 & 0 & 0 & 0 & 2 \\
\hline conocimiento frente & Principiante & 3 & 10 & 3 & 0 & 0 & 16 \\
\hline \multirow[t]{2}{*}{ al uso de las TIC } & Intermedio & 0 & 1 & 5 & 4 & 1 & 11 \\
\hline & Avanzado & 0 & 0 & 0 & 0 & 1 & 1 \\
\hline Total & & 3 & 13 & 8 & 4 & 2 & 30 \\
\hline
\end{tabular}




\begin{tabular}{lccr}
\hline & Valor & gl & Sig. asintótica (bilateral) \\
\hline Chi-cuadrado de Pearson & $32,778^{\mathrm{a}}$ & 12 & 0,001 \\
Razón de verosimilitudes & 28,601 & 12 & 0,005 \\
Asociación lineal por lineal & 15,332 & 1 & 0,000 \\
N de casos válidos & 30 & & \\
\hline
\end{tabular}

a. 19 casillas $(95,0 \%)$ tienen una frecuencia esperada inferior a 5 . La frecuencia mínima esperada es $0,07$.

Fuente: Encuesta.

Después de realizar el análisis descriptivo Chi-cuadrado de Pearson, en la tabla 4 se demuestra una relación entre las variables de formación de la población y el conocimiento sobre el uso de las TIC, puesto que, la significación asintótica bilateral tiene un valor de 0,001 inferior a 0,005 , asumiendo dicha relación. Lo que quiere decir, que hay una carencia de formación y por ende, una falta de conocimiento sobre cómo usar las TIC.

\section{Tabla 4.}

Estadísticos descriptivos.

\begin{tabular}{llllrrr}
\hline & $N$ & Mínimo & Máximo & Media & Desv. típ. \\
\hline Falta de acceso a internet & 30 & 1 & 3 & 1,23 & 0,504 \\
Falta de competencias digitales & 30 & 3 & 5 & 3,73 & 0,583 \\
\hline N válido (según lista) & 30 & & & & & \\
\hline
\end{tabular}

Fuente: Encuesta.

Con respecto a la variable de acceso a la tecnología, tenemos que, el promedio es de 1,23 y con respecto a la falta de competencias digitales es de 3,73. Lo que significa que la población analizada tiene más dificultad en usar la tecnología que en acceder a ella. Por lo tanto, se evidencia que existe una brecha digital de uso, debido a que la falta de competencias digitales fue un factor que impidió a los padres de familia colaborar en gran porcentaje en la educación de sus hijos de nivel inicial. 
Para obtener los resultados cualitativos de la investigación se aplicó el grupo focal, considerando una muestra de 6 padres de familia del nivel inicial de la escuela Mercedes Vázquez Correa del cantón Gualaceo. Lo primero que se realizó fue programar una reunión en la plataforma zoom con un tiempo de 40 minutos, luego se dio inicio con un diálogo introductorio y finalmente se continuó con las preguntas que se iban contestando mediante un conversatorio entre la moderadora y los entrevistados. El objetivo fue conseguir las percepciones acerca el tema y llegar a una mejor comprensión que pueda apoyar la investigación. 


\section{Tabla 5.}

Resultados cualitativos.

\begin{tabular}{|c|c|c|}
\hline Unidad de análisis & Categoria & Segmento \\
\hline $\begin{array}{l}\text { Tiempo } \\
\text { dedicación }\end{array}$ & $\begin{array}{l}\text { Tiempo de dedicación } \\
\text { para ayudar en las } \\
\text { actividades escolares }\end{array}$ & $\begin{array}{l}\text { - Todo el día porque ellos son niños pequeños y se merecen } \\
\text { todo el tiempo del día para ayudarlos. } \\
\text { - Dedicación totalmente porque son pequeños } \\
\text { - La dedicación es bastante, pues necesitan todo el apoyo } \\
\text { porque recién están iniciando en la educación. }\end{array}$ \\
\hline sabilidad & $\begin{array}{lr}\text { Existe } & \text { más } \\
\text { responsabilidad } & y \\
\text { acompañamiento a los } \\
\text { niños de inicial }\end{array}$ & $\begin{array}{l}\text { - Hay más responsabilidad porque los niños son pequeños } \\
\text { y no saben mucho del uso de la tecnología. } \\
\text { - Hay que estar pendientes de los niños su edad y } \\
\text { aprendizaje. } \\
\text { - La responsabilidad es constante porque son niños de } 4 \\
\text { años, pues es muy diferente con los hijos más grandes, } \\
\text { pues ellos pueden hacer las cosas más } \\
\text { independientemente, conectarse a las clases solos, en } \\
\text { cambio los niños pequeños no, por eso hay que } \\
\text { comprometerse más. }\end{array}$ \\
\hline Capacitación & $\begin{array}{lr}\text { Conocimiento de } \\
\text { herramientas } & \text { y } \\
\text { plataformas digitales } & \end{array}$ & $\begin{array}{l}\text { - En realidad no hemos podido capacitarnos, por la falta de } \\
\text { tiempo } \\
\text { - No se ha tenido ninguna capacitación debido al tiempo y } \\
\text { al trabajo. } \\
\text { - No me he capacitado, por falta de coordinación y } \\
\text { oportunidades }\end{array}$ \\
\hline Brecha digital & $\begin{array}{l}\text { El desconocimiento del } \\
\text { uso de la tecnología } \\
\text { causa una brecha digital }\end{array}$ & $\begin{array}{l}\text { - Sí, la tecnología es algo nuevo para los padres de familia, } \\
\text { en nuestra época no se aplicaba, por lo que, no tenemos } \\
\text { mucho conocimiento y ahora nos ha afectado mucho. } \\
\text { - El desconocimiento nos perjudica porque si no sabemos } \\
\text { nosotros, nos quedamos trruncados, fuera bueno que a } \\
\text { nosotros también tengamos ayudada para capacitarnos } \\
\text { para poder ayudar a nuestros hijos. }\end{array}$ \\
\hline
\end{tabular}


- El inexperiencia evita que prosperemos, porque si nosotros no sabemos tampoco podemos ayudar a nuetsros hijos, así ellos nos pidan ayuda no podemos ayudar porque no sabemos.

\begin{tabular}{|c|c|c|}
\hline Capacitación & $\begin{array}{l}\text { Capacitaciones sobre el } \\
\text { uso de la tecnología }\end{array}$ & $\begin{array}{l}\text { - Fuera bueno que ha nosotros también nos capacitaran o } \\
\text { enseñaran, o hubieran lugares que pudieran capacitarnos. } \\
\text { - Para mi pensar, los niños son pequeños y es necesario } \\
\text { que desde ya les vayamos formando y si los padres no } \\
\text { estamos preparados no podemos dar la ayuda necesaria a } \\
\text { nuestros niños. } \\
\text { - Mientras los padres no sepamos ayudarlos, ellos también } \\
\text { van a quedarse ahí. } \\
\text { - La tecnología va a ser el futuro, si no nos ayudan con } \\
\text { alguna capacitación nos estamos quedando atrás, no } \\
\text { estamos avanzando en la educación. } \\
\text { - Sería una buena idea unas capacitaciones constantes } \\
\text { para poder ayudar a nuestros hijos. } \\
\text { - Aunque el tiempo es una dificultad, los padres de familia } \\
\text { nos diéramos un tiempito, ya que sería el beneficio para mí } \\
\text { y para mis hijos. es por eso. } \\
\text { - Solo deberíamos coordinar los tiempos, ya que sería un } \\
\text { tiempo valioso y estaríamos invirtiendo en nuestros hijos. }\end{array}$ \\
\hline
\end{tabular}

Fuente: Encuesta.

El rol de los padres de familia o representantes legales es muy importante en la educación de los estudiantes y se ha evidenciado aún más en la educación virtual en tiempos de pandemia, debido a que, fueron ellos que a pesar de no estar capacitados o sin algún conocimiento sobre la tecnología asumieron los retos y dificultades presentadas para poder ayudar a sus representados.

Cabe resaltar el rol importante del docente en las clases, sin embargo, es también valiosa la participación del padre de familia, puesto que es el primer aliado para hacer llegar el 
aprendizaje al niño, sobretodo en el nivel inicial, debido a que son niños de 3 y 4 años de edad que necesitan la ayuda, colaboración, coordinación necesaria y responsable en equipo entre docentes y representantes.

Es por esta razón, que a partir del análisis se puede demostrar que las percepciones de los padres de familia están ligadas a la falta de competencias, conocimiento y uso de la tecnología como una brecha digital en la educación de los estudiantes del nivel inicial, que no permite avanzar satisfactoriamente, debido a que son los representantes los que colaboran directamente en su aprendizaje. Por este motivo, al tener un rol importante, deben tener un conocimiento acorde o favorecedor para poder guiar el desarrollo del aprendizaje en sus hogares.

\section{PROPUESTA}

Los resultados de la presente investigación demuestran que la falta de conocimiento de padres de familia sobre el uso de la tecnología se ha convertido en un inconveniente para poder guiar el aprendizaje de sus hijos del subnivel inicial en tiempos de pandemia. Es por este motivo, que es conveniente tomar en cuenta la capacitación como primordial actividad de las instituciones y sobre todo de las entidades locales como el municipio o el distrito de educación.

Cabe mencionar, que el rol del docente como educador es muy enriquecedor e irremplazable, pero también se debe tomar en cuenta el apoyo directo que los padres de familia tienen y han demostrado a partir de la pandemia. Es decir, debido a los efectos de la pandemia, la educación continuó desde los hogares, obligando a los docentes dictar sus clases online y aplicar recursos, herramientas y utilizar diariamente dispositivos electrónicos. Por supuesto, que a los estudiantes se les hace más fácil adaptarse a la nueva forma de educar que sus docentes aplican por ser nativos digitales, pero no se toma en cuenta que los padres de familia también se ven obligados a la nueva educación y a ellos complicándose más por ser migrantes digitales. 
Por esa razón, la propuesta que a continuación se presenta consiste en un plan de capacitación sobre el uso de recursos, plataformas, y herramientas digitales básicas, en el contexto de la educación virtual, pero que incluya a los actores principales de la educación, docente y padres de familia para que pueda llegar el aprendizaje adecuado a los estudiantes. Esto ayudará a trabajar de manera conjunta y se podrá obtener resultados favorecedores para la educación. Es por eso, que para la propuesta se reflexionó sobre una triada de capacitación que se expone en el siguiente gráfico.

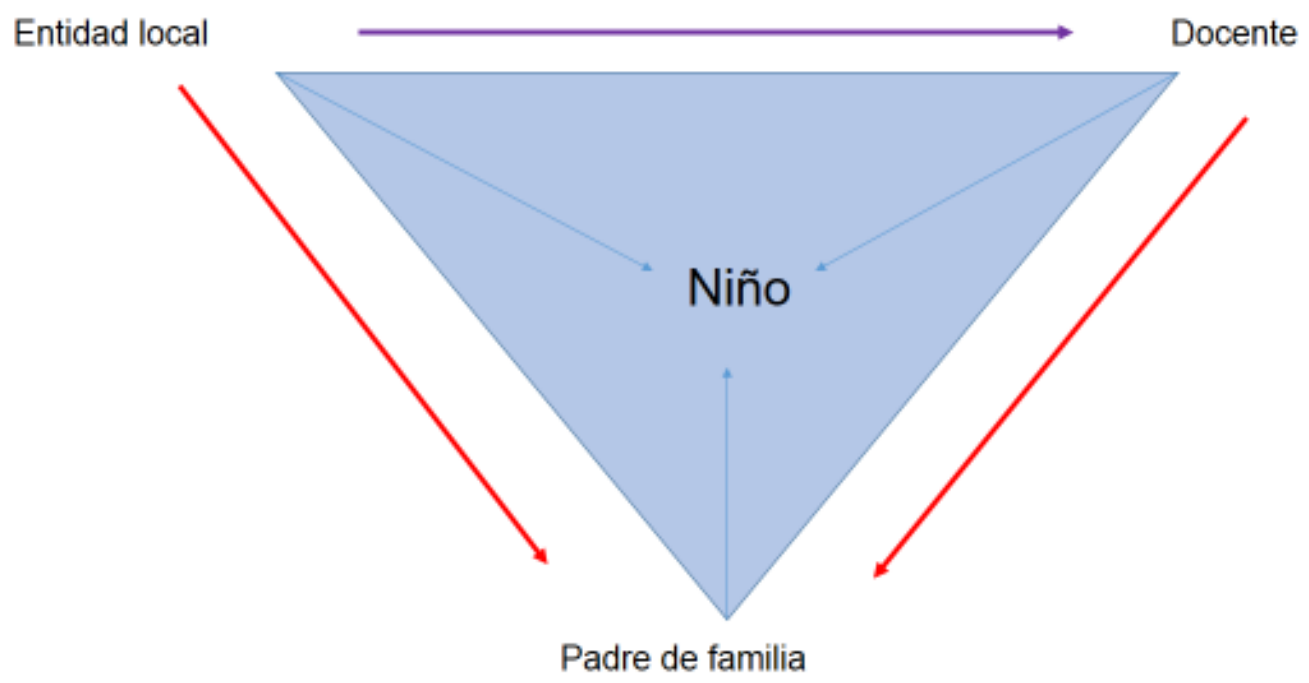

Figura 1. Triada de capacitación.

Elaboración: Los autores.

A continuación, se explica detalladamente la propuesta de capacitación.

Entidad local: Gualaceo es un cantón que ha apoyado a sus habitantes con diferentes capacitaciones en el área comercial, pero no se ha impulsado capacitaciones en el campo educativo, por lo que, es importante para todos los sujetos educativos talleres que generen unidad, igualdad, privilegios y oportunidades de aprender constantemente sobre 
educación y tecnología, para de esta manera avanzar en el desarrollo del aprendizaje y así estar preparados para alguna emergencia como lo fue la pandemia. Además, una ayuda oportuna es también capacitaciones por parte del Distrito de educación no solo a docentes o directivos, sino también a padres de familia y a estudiantes, en diferentes modalidades, horarios y días flexibles para que no se interrumpan horas académicas y laborables, ya que, de esta manera, la información llegaría de manera directa. Esto favorecerá para que todos los sujetos educativos permanezcan en constante preparación y la educación pueda avanzar satisfactoriamente y de manera conjunta.

Docente: El trabajo del docente ha sido valioso en la actualidad, ya que ha tenido que capacitarse por cualquier medio para poder impartir sus clases virtuales como cursos, talleres, guías, videos tutoriales sobre el uso de recursos, herramientas digitales, sobre todo las plataformas básicas y gratuitas que han sido de gran utilidad, como padlet, Genially, Kahoot, Wordwall, Youtube, y que se han implementado para la enseñanza aprendizaje de sus alumnos. Por lo tanto, el docente puede impartir una capacitación corta y concisa sobre lo que se va a utilizar en las clases con sus hijos, para que tengan conocimiento y no haya complicaciones al colaborar. Esta capacitación consta de dos momentos, la una a iniciar el primer quimestre y la otra en el segundo quimestre, de manera virtual para que haya la asistencia total de los representantes legales.

Finalmente, la triada de capacitación consiste en aprender y trabajar de manera conjunta, con la ayuda de entidades locales como del docente para poder enseñar al padre de familia y guiarlo para que esté preparado y pueda ayudar en el aprendizaje de su hijo del subnivel inicial, debido a que son niños que necesitan la colaboración del docente como de su familia. 


\section{CONCLUSIONES}

La investigación tuvo como objetivo indagar sobre las percepciones de los padres de familia acerca las dificultades obtenidas en el acompañamiento de sus hijos del subnivel inicial. Actualmente, es este tiempo de pandemia es muy importante conocer los problemas que se presentan y que está causando desigualdad en la educación, por lo que, surgió el interés por descubrir si los padres de familia tienen conocimiento en tecnología, eje principal de la educación virtual.

Con los resultados cualitativos obtenidos, se evidenció que el principal problema en la escuela Mercedes Vázquez Correa es la brecha digital de uso de la tecnología, pues existe un promedio de 1,23 que no tienen dificultad en acceder a la tecnología, en cambio un promedio de 3,73 tiene dificultad en usarla a causa de la falta de conocimiento.

De igual manera, con el resultado cualitativo se pudo conocer de cerca las perspectivas de los padres de familia, donde demostró que entre los retos que tuvieron que enfrentar fue saber usar la tecnología, ya que tienen un nivel bajo en competencias digitales que les impide ayudar en su totalidad a sus hijos de inicial, puesto que son niños de 3 y 4 años que requieren el acompañamiento constante tanto en las clases sincrónicas y asincrónicas, lo que muchos tuvieron que acoplarse de la mejor manera posible.

Es más, los padres de familia se sienten preocupados por no estar preparados, mencionan que es necesario que alguna autoridad les ayude, ya que, la tecnología vino a quedarse y seguirá avanzando y si ellos no están capacitados truncará también la educación de sus hijos y eso atrasará su instrucción.

La propuesta presentada pretende trabajar de manera conjunta con toda la comunidad educativa, mediante capacitaciones que permitan formar en conocimientos y usos de las TIC, esto mediante la colaboración de entidades locales como el Gad Municipal y el Distrito de educación de Gualaceo. Además, la ayuda del docente en aula de clases, con la capacitación sobre el plan de estudio que se desarrollará durante el quimestre. Un trabajo duro pero que tendrá su recompensa, pues los padres de familia son un apoyo esencial en los hogares para fortalecer el aprendizaje de los niños. 


\section{FINANCIAMIENTO}

No monetario.

\section{AGRADECIMIENTO}

A la Corporación Eléctrica del Ecuador y la Jefatura de Posgrados de la Universidad Católica de Cuenca por permitir el desarrollo y fomento de la investigación.

\section{REFERENCIAS CONSULTADAS}

Alpizar, G., Biehl, L., Hernández-Agramonte, J., Namen, O., Näslund-Hadley, E., Ochoa, L., \& Peña, B. (2020). El involucramiento de padres en la educación a distancia y la brecha de género en salud mental [Parental involvement in distance education and the gender gap in mental health]. https://n9.cl/gy0l

Casas-Anguita, J., Repullo Labrador, J. R., \& Donado Campos, J. (2003). La encuesta como técnica de investigación. Elaboración de cuestionarios y tratamiento estadístico de los datos (II) [Surveys as a research technique. Composition of questionnaires and statistical processing of data (II)]. Atencion primaria, 31(9), 592-600. https://doi.org/10.1016/s0212-6567(03)79222-1

Castillo-Fuerman, M. (2020). Los retos de la Educación Inicial en tiempos de COVID-19 [The challenges of Early Childhood Education in times of COVID-19]. https://n9.cl/dj9af

El Comercio. (2020). Pandemia aumentó riesgo de abandono escolar en Ecuador [Pandemic increased risk of school dropout in Ecuador]. https://n9.cl/in5u7

Gunkel, D. (2003). Second thoughts: toward a critique of the digital divide [Segundos pensamientos: hacia una crítica de la brecha digital]. new media \& society, 5(4), 499-522. https://n9.cl//z7b7

Martín-Romero, A. M. (2020). La brecha digital generacional [The generational digital divide]. Revista Andaluza de Trabajo y Bienestar Social, 151, 77-93. https://n9.cl/bxo1c

Ministerio de Educación MINEDUC. (2020). Acuerdo Nro.-2020-00013-A [Agreement No.-2020-00013-A]. https://n9.cl/7hdrt 
Ministerio de Telecomunicaciones y de la Sociedad de la Información, MINTEL. (2019). Libro Blanco de Territorios Digitales en Ecuador [White Paper on Digital Territories in Ecuador]. https://n9.cl/zbifv

Ministerio de Telecomunicaciones y de la Sociedad de la Información MINTEL. (2020). Avances en TIC para acceder a más conectividad [Advances in TIC to access more connectivity]. https://n9.cl/gycf

Mosquera, X. (2020). La brecha digital: su impacto en la educación en línea [The digital divide: its impact on online education]. https://n9.cl/5vp1w

Organización de las Naciones Unidas para la Educación, la Ciencia y la Cultura, UNESCO. (2020a). Objetivo 4: Garantizar una educación inclusiva, equitativa y de calidad y promover oportunidades de aprendizaje durante toda la vida para todos [Goal 4: Ensure inclusive, equitable and quality education and promote lifelong learning opportunities for all]. https://n9.cl/w4ma

Organización de las Naciones Unidas para la Educación, la Ciencia y la Cultura, UNESCO. (2018). Las competencias digitales son esenciales para el empleo y la inclusión social [Digital skills are essential for employment and social inclusion]. https://n9.cl/te11

Organización de las Naciones Unidas para la Educación, la Ciencia y la Cultura, UNESCO. (2020b). Surgen alarmantes brechas digitales en el aprendizaje a distancia [Alarming digital gaps emerge in distance learning]. https://n9.cl/10lau

Quiroz-Reyes, C. (2020). Pandemia Covid-19 e Inequidad Territorial: El Agravamiento de las Desigualdades Educativas en Chile [Covid-19 Pandemic and Territorial Inequity: The Worsening of Educational Inequalities in Chile]. Revista Internacional De Educación Para La Justicia Social, 9(3).

Rodicio-García, M. L., Ríos-de-Deus, M. P., Mosquera-González, M. J., \& Penado Abilleira, M. . (2020). La Brecha Digital en Estudiantes Españoles ante la Crisis de la Covid-19 [The Digital Divide in Spanish Students in the Face of the Covid-19 Crisis]. Revista Internacional De Educación Para La Justicia Social, 9(3), 103-125. https://doi.org/10.15366/riejs2020.9.3.006

Rodríguez-Gallardo, A. (2006). La brecha digital y sus determinantes [The digital divide and its determinants]. https://n9.cl/gxv1 
EPISTEME KOINONIA

Revista Electrónica de Ciencias de la Educación, Humanidades, Artes y Bellas Artes

Año IV. Vol IV. Nº 8 . Julio - Diciembre. 2021

Hecho el depósito de Ley: FA2018000022

ISSN: $2665-0282$

FUNDACIÓN KOINONIA (F.K).

Santa Ana de Coro, Venezuela

Diana Gabriela Bermeo-Chalco; Darwin Gabriel García-Herrera; Sandra Elizabeth Mena-Clerque

Stingl, A.I. (2016) Digital Divide. In: ten Have H. (eds) Encyclopedia of Global Bioethics. Springer, Cham. https://doi.org/10.1007/978-3-319-09483-0 246

Warschauer, M. (2001). A literacy approach to the digital divide. https://n9.cl/cs1di

C2021 por los autores. Este artículo es de acceso abierto y distribuido según los términos y condiciones de la licencia Creative Commons Atribución-NoComercial-Compartirlgual 4.0 Internacional (CC BY-NC-SA 4.0) (https://creativecommons.org/licenses/by-nc-sa/4.0/). 